\title{
Novel Monte Carlo algorithms and their applications
}

\author{
Yutaka Okabe $^{1}$, Yusuke Tomita, Chiaki Yamaguchi \\ Department of Physics, Tokyo Metropolitan University, Hachioji, Tokyo \\ 192-0397, Japan
}

\begin{abstract}
We describe a generalized scheme for the probability-changing cluster (PCC) algorithm, based on the study of the finite-size scaling property of the correlation ratio, the ratio of the correlation functions with different distances. We apply this generalized PCC algorithm to the two-dimensional 6-state clock model. We also discuss the combination of the cluster algorithm and the extended ensemble method. We derive a rigorous broad histogram relation for the bond number. A Monte Carlo dynamics based on the number of potential moves for the bond number is proposed, and applied to the three-dimensional Ising and 3-state Potts models.
\end{abstract}

Key words: Cluster algorithm; Finite-size scaling; Correlation ratio; Broad histogram relation

\section{Introduction}

In the Monte Carlo simulation, we sometimes suffer from the problem of slow dynamics. The critical slowing down near the critical point, the phase separation dynamics at low temperature, the slow dynamics due to the randomness or frustration, and the low-temperature slow dynamics in quantum Monte Carlo simulation are examples of the problems of slow dynamics.

We may classify the attempts to conquer the slow dynamics in the Monte Carlo simulation into two categories. The first category is the cluster algorithm, such as the Swendsen-Wang (SW) algorithm [1] and the Wolff algorithm [2]. The second one is the extended ensemble method. The multicanonical method $[3,4]$, the broad histogram method [5], and the flat histogram method $[6,7]$ are

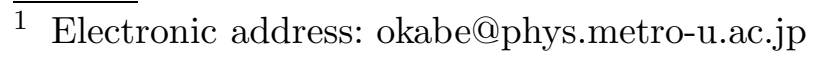

Preprint submitted to Elsevier Science 
examples of the second category. Recently, Wang and Landau [8] proposed an efficient algorithm to accelerate the calculation of the energy density of states (DOS). Yamaguchi and Okabe [9] have successfully used the Wang-Landau algorithm for the study of the antiferromagnetic $q$-state Potts models.

Tomita and Okabe [10] recently proposed an effective cluster algorithm, which is called the probability-changing cluster (PCC) algorithm, of tuning the critical point automatically. The PCC algorithm is an extension of the SW algorithm [1]; we change the probability of connecting spins of the same type, essentially the temperature, in the process of the Monte Carlo spin update. We showed the effectiveness of the PCC algorithm for the two-dimensional (2D) and three-dimensional (3D) Potts models [10], determining the critical point and exponents. We can extract information on critical phenomena with much less numerical effort. The PCC algorithm is quite useful for studying random systems, where the distribution of the critical temperature, $T_{c}$, is important. We applied the PCC algorithm to the 2D diluted Ising model [11], investigating the crossover and self-averaging properties of random systems. We also extended the PCC algorithm to the problem of the vector order parameter [12]; studying the $2 \mathrm{D}$ XY and clock models, we showed that the PCC algorithm is also useful for the Kosterlitz-Thouless (KT) transition [13].

The combination of approaches of two categories, the cluster algorithm and the extended ensemble method, is a challenging problem. Janke and Kappler [14] proposed a trial to combine the multicanonical method and the cluster algorithm; their method is called the multibondic ensemble method. Recently, Yamaguchi and Kawashima [15] improved the multibondic ensemble method, and also showed that the combination of the Wang-Landau algorithm and the improved multibondic ensemble method yields much better statistics compared to the original multibondic ensemble method [14].

Here, we pick up two recent topics of new Monte Carlo algorithms. We first discuss the generalization of the PCC algorithm. This generalized scheme is based on the finite-size scaling (FSS) property of the correlation ratio. Second, for the algorithm to combine the cluster algorithm and the extended ensemble method, we derive a rigorous broad histogram relation for the bond number, and propose the flat histogram method for the bond number.

\section{Generalized scheme for the PCC algorithm}

We start with reviewing the idea of the PCC algorithm [10]. We explain the case of the ferromagnetic $q$-state Potts model, whose Hamiltonian is given by

$$
\mathcal{H}=-J \sum_{<i, j>}\left(\delta_{\sigma_{i}, \sigma_{j}}-1\right), \quad \sigma_{i}=1,2, \cdots, q .
$$


We construct the Kasteleyn and Fortuin (KF) [16] clusters using the probability $p$, where $p$ is the probability of connecting spins of the same type, $p=1-e^{-J / k_{B} T}$. The correspondence of the spontaneous magnetization of the $q$-state Potts model and the percolation probability of the bond percolation model was discussed by $\mathrm{Hu}$ [17]. Then, we check whether the system is percolating or not. If the system is percolating (not percolating) in the previous test, we decrease (increase) $p$ by $\Delta p(>0)$. Spins are updated following the same rule as the SW algorithm. After repeating the above processes, the distribution of $p$ for Monte Carlo samples approaches the Gaussian distribution of which mean value is $p_{c}(L) ; p_{c}(L)$ is the probability of connecting spins, such that the existence probability $E_{p}$ becomes $1 / 2$. The existence probability $E_{p}$ [18] is the probability that the system percolates. Since $E_{p}$ follows the FSS near the critical point,

$$
E_{p}(p, L) \sim X\left(t L^{1 / \nu}\right), \quad t=\left(p_{c}-p\right) / p_{c}
$$

where $p_{c}$ is the critical value of $p$ for the infinite system and $\nu$ is the correlationlength critical exponent, we can estimate $p_{c}$ from the size dependence of $p_{c}(L)$ using Eq. (2) and, in turn, estimate $T_{c}$ through the relation $p_{c}=1-e^{-J / k_{B} T_{c}}$.

In the original formulation of the PCC algorithm [10], we used the KF representation in two ways. First, we make a cluster flip as in the SW algorithm [1]. Second, we change the probability of connecting spins of the same type, $p$, depending on the observation whether clusters are percolating or not. The point is that $E_{p}$ has the FSS property with a single scaling variable. We may use quantities other than $E_{p}$ which have a similar FSS relation. In the FSS analysis of the Monte Carlo simulation, we often use the Binder ratio [19], which is essentially the ratio of the moments of the order parameter $m$. The moment ratio has the FSS property with a single scaling variable,

$$
\frac{\left\langle m^{4}\right\rangle}{\left\langle m^{2}\right\rangle^{2}}=f\left(t L^{1 / \nu}\right)
$$

as far as the corrections to FSS are negligible. The angular brackets indicate the thermal average. The moment ratio derived from a snapshot spin configuration is always one; therefore, the instantaneous moment ratio cannot be used for the criterion of judgment whether we increase or decrease the temperature.

As another quantity, we may treat the correlation ratio, the ratio of the correlation functions with different distances. For an infinite system at the critical point, the correlation function decays as a power of $r$,

$$
\langle g(r)\rangle \sim r^{-(D-2+\eta)}, \quad(L=\infty, t=0)
$$


with the decay exponent $\eta$. Precisely, the distance $r$ is a vector, but we have used a simplified notation. Away from the critical point, the ratio of the distance $r$ and the correlation length $\xi$ plays a role in the scaling of the correlation function. Moreover, for finite systems, two length ratios, $r / L$ and $L / \xi$, come in the scaling form of the correlation function. Then, the ratio of the spin-spin correlation functions with different distances $r$ and $r^{\prime}$ takes the FSS form with a single scaling variable,

$$
\frac{\langle g(r, t, L)\rangle}{\left\langle g\left(r^{\prime}, t, L\right)\right\rangle}=\tilde{f}(L / \xi),
$$

if we fix two ratios, $r / L$ and $r / r^{\prime}$. In case the correlation length diverges with a power law, $\xi \propto t^{-\nu}$, Eq. (5) becomes the same form as Eq. (3); however, Eq. (5) is also applicable to the case of the KT transition, where the correlation length diverges more strongly than the power-law divergence.

In order to examine the FSS properties of the correlation ratio, we here give the result of the 2D 6-state clock model on the square lattice with the periodic boundary conditions. The $2 \mathrm{D} q$-state clock model is known to exhibit two phase transitions of the KT type at $T_{1}$ and $T_{2}\left(T_{1}<T_{2}\right)$ for $q>4$ [20]. We simulate the 2D 6-state clock model by the use of the Wang-Landau algorithm [8]. We show the temperature dependence of both the moment ratio $\left\langle m^{4}\right\rangle /\left\langle m^{2}\right\rangle^{2}$ and the correlation ratio $\langle g(L / 2)\rangle /\langle g(L / 4)\rangle$ in Fig. 1. As for the distances $r$ and $r^{\prime}$, we choose $L / 2$ and $L / 4$; we take the horizontal or vertical direction of the lattice for the orientation of two sites. For the correlation ratio, the curves of different sizes merge in the intermediate $\mathrm{KT}$ phase $\left(T_{1}<T<T_{2}\right)$, and spray out for the low-temperature ordered and high-temperature disordered phases, which is expected from the FSS form of Eq. (5). Then, we can make a FSS analysis based on the KT form of the correlation length,

$$
\xi \propto \exp (c / \sqrt{t})
$$

where $t=\left|T-T_{\mathrm{KT}}\right| / T_{\mathrm{KT}}$. Using the data of $\langle g(L / 2)\rangle /\langle g(L / 4)\rangle$ for $L=12,16$, $24,32,48$, and 64 , we estimate two KT transition temperatures. The bestfitted estimates are $T_{1}=0.698(4)$ and $T_{2}=0.898(4)$, in units of $J / k_{B}$, which are compatible with the recent results using the PCC algorithm [12], $T_{1}=0.7014(11)$ and $T_{2}=0.9008(6)$. On the contrary, as seen from Fig. 1, the corrections to FSS are larger for the moment ratio, which makes the FSS analysis difficult. We have shown that the correlation ratio is a good estimator especially for the $\mathrm{KT}$ transition. It is due to the fact that we only use the property of correlation function; the characteristic of the KT transition is that the correlation function shows a power-law decay at all the temperatures of the KT phase.

We may use the FSS properties of the correlation ratio for the generalization of the PCC algorithm. Instead of checking whether the clusters are percolating 


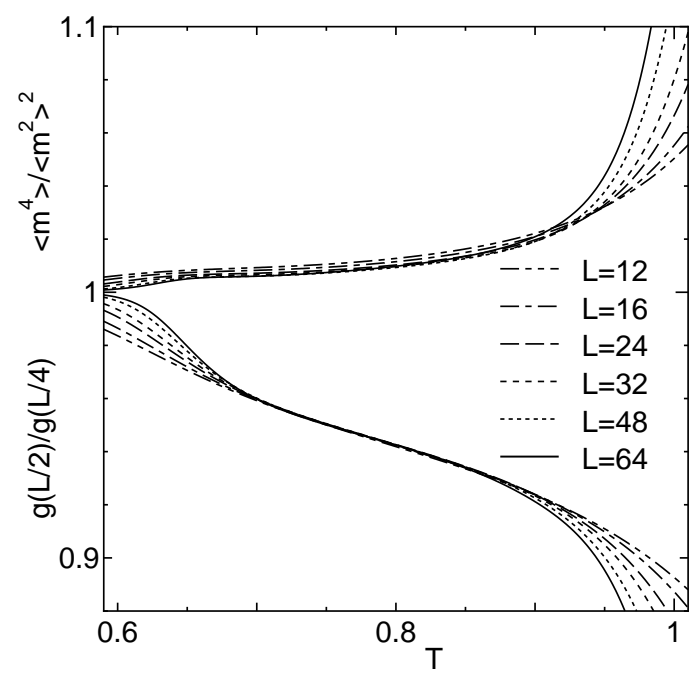

Fig. 1. The moment ratio $\left\langle m^{4}\right\rangle /\left\langle m^{2}\right\rangle^{2}$ and the correlation ratio $\langle g(L / 2)\rangle /\langle g(L / 4)\rangle$ of the 2D 6-state clock model for $L=12,16,24,32,48$, and 64 .

or not, we ask whether the instantaneous correlation ratio $g(L / 2) / g(L / 4)$ is larger or smaller than some fixed value $R_{c}$. Of course, we can use other sets of distances. We decrease (increase) the temperature, if $g(L / 2) / g(L / 4)$ is smaller (larger) than $R_{c}$. We start the simulation with some temperature. We make the amount of the change of temperature, $\Delta T$, smaller during the simulation; in the limit of $\Delta T \rightarrow 0$, the system approaches the canonical ensemble.

As an example, we apply the generalized scheme of the PCC algorithm to the study of the 2D 6-state clock model. We treat the systems with linear sizes $L=8,16,32,64$, and 128 . We start with $\Delta T=0.005$, and gradually decrease $\Delta T$ to the final value, 0.0001. After 20,000 Monte Carlo sweeps of determining $T_{\mathrm{KT}}(L)$, we make 10,000 Monte Carlo sweeps to take thermal average; we make 20 runs for each size to get better statistics and to evaluate the statistical errors. We calculate $g(L / 2) / g(L / 4)$ to check whether it is larger than $R_{c}$ or not.

We use the FSS analysis based on the KT form of the correlation length, Eq. (6). For the $L$ dependence of $T_{\mathrm{KT}}(L)$, we have the relation

$$
T_{\mathrm{KT}}(L)=T_{\mathrm{KT}}+\frac{c^{2} T_{\mathrm{KT}}}{(\ln b L)^{2}}
$$

We plot $T_{2}(L)$ as a function of $l^{-2}$ with $l=\ln b L$ for the best-fitted parameters in Fig. 2(a). Here, we concentrate on the high-temperature transition $T_{2}(L)$. The value of $R_{c}$ has been set to be 0.87 and 0.85 . The error bars are smaller than the size of marks. We should mention that the data with different $R_{c}$ are collapsed on a single curve in this plot, which means that $b$ depends on $R_{c}$ in Eq. (7) and the difference of $R_{c}$ can be absorbed in the $R_{c}$ dependence 

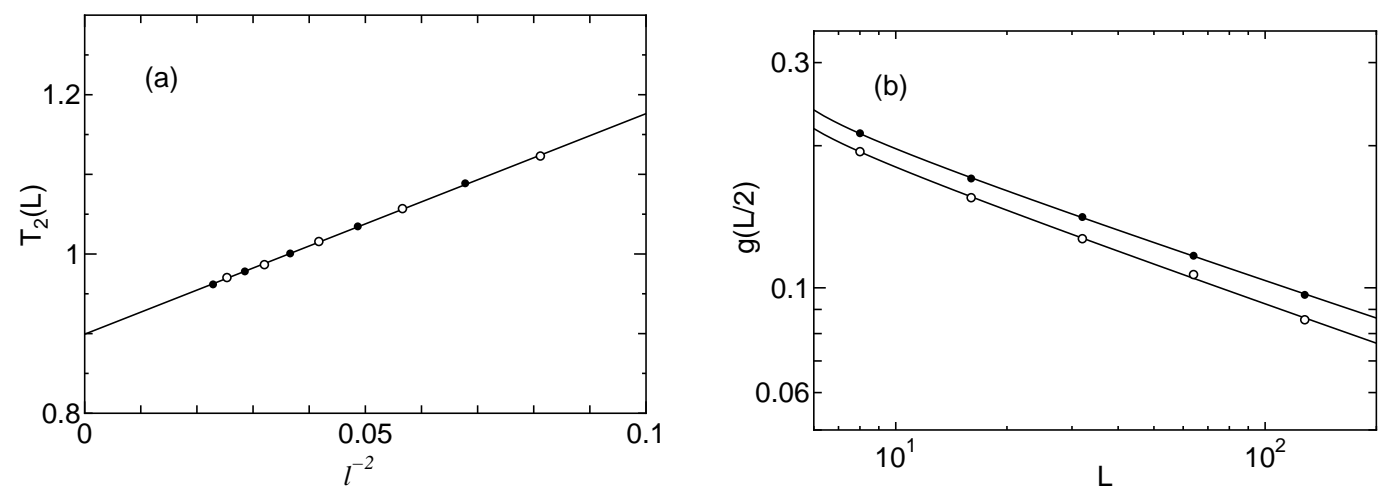

Fig. 2. (a) Plot of $T_{2}(L)$ and (b) the logarithmic plots of $\langle g(L / 2)\rangle$ at $T_{2}(L)$ of the 2D 6 -state clock model for $L=8,16,32,64$, and 128 , where $l=\ln b L$. The closed circles are data for $R_{c}=0.87$, and open circles are those for $R_{c}=0.85$.

of $b$. The present estimate of $T_{2}$ is $0.899(3)$. This value is consistent with the estimate of the PCC algorithm using the percolating properties, 0.9008(6) [12].

We also estimate the critical exponent $\eta$ from the size dependence of the correlation at $T_{2}(L)$. In Fig. 2(b), we plot $\langle g(L / 2)\rangle$ at $T_{2}(L)$ as a function of $L$ in a logarithmic scale. For the estimate of $\eta$, we use the FSS form including small multiplicative logarithmic corrections

$$
\langle g(L / 2)\rangle=A L^{-\eta}\left(\ln b^{\prime} L\right)^{-2 r}
$$

Our estimate is $\eta=0.250$ (3) from the data for $R_{c}=0.87$, and $\eta=0.265(2)$ from the data for $R_{c}=0.85$, which are compatible with the theoretical prediction, $1 / 4(=0.25)$.

The detailed description of the generalized scheme of the PCC algorithm based on the FSS of the correlation ratio together with its application to the $2 \mathrm{D}$ quantum XY model of $S=1 / 2$ will be reported elsewhere [21].

\section{Cluster-flip flat histogram method}

In this section, we consider the combination of the cluster algorithm and the extended ensemble method. One calculates the energy DOS, $g(E)$, in the multicanonical method [3,4] and the Wang-Landau method [8]; the energy histogram $H(E)$ is checked during the Monte Carlo process. In contrast, the DOS for bond number $n_{b}, \Omega\left(n_{b}\right)$, is calculated in the multibondic ensemble method [14] or its improvement by Yamaguchi and Kawashima [15]; the histogram for bond number, $H\left(n_{b}\right)$, is checked in the Monte Carlo process.

In proposing the broad histogram method, Oliveira et al. [5] paid attention to 
the number of potential moves, or the number of the possible energy change, $N\left(S, E \rightarrow E^{\prime}\right)$, for a given state $S$. The total number of moves is

$$
\sum_{\Delta E} N(S, E \rightarrow E+\Delta E)=N
$$

for a single-spin flip process, where $N$ is the number of spins. The energy DOS is related to the number of potential moves as

$$
g(E)\left\langle N\left(S, E \rightarrow E^{\prime}\right)\right\rangle_{E}=g\left(E^{\prime}\right)\left\langle N\left(S^{\prime}, E^{\prime} \rightarrow E\right)\right\rangle_{E^{\prime}},
$$

where $\langle\cdots\rangle_{E}$ denotes the microcanonical average with fixed $E$. This relation is shown to be valid on general grounds [22,23], and hereafter we call Eq. (10) as the broad histogram relation (BHR) for the energy. One may use the number of potential moves $N\left(S, E \rightarrow E^{\prime}\right)$ for the probability of updating states. Alternatively, one may employ other dynamics which has no relation to $N\left(S, E \rightarrow E^{\prime}\right)$, but Eq. (10) is used when calculating the energy DOS.

It is interesting to ask whether there is a relation similar to the BHR, Eq. (10), for the bond number. Here, using the cluster (graph) representation, we derive the BHR for the bond number. We consider the $q$-state Potts model as an example. With the framework of the dual algorithm [24,25], the partition function is expressed in the double summation over state $S$ and graph $G$ as

$$
Z(T)=\sum_{S, G} V_{0}(G) \Delta(S, G)
$$

where $\Delta(S, G)$ is a function that takes the value one when $S$ is compatible to $G$ and takes the value zero otherwise. A graph consists of a set of bonds. The weight for graph $G, V_{0}(G)$, is defined as

$$
V_{0}(G)=V_{0}\left(n_{b}(G), T\right)=\left(e^{J / k_{B} T}-1\right)^{n_{b}(G)}
$$

for the $q$-state Potts model, where $n_{b}(G)$ is the number of "active" bonds in $G$. We say a pair $(i, j)$ is satisfied if $\sigma_{i}=\sigma_{j}$, and unsatisfied otherwise. Satisfied pairs become active with a probability $p=1-e^{-J / k_{B} T}$ for given $T$.

By taking the summation over $S$ and $G$ with fixing the number of bonds $n_{b}$, the expression for the partition function becomes

$$
Z(T)=\sum_{n_{b}=0}^{N_{B}} \Omega\left(n_{b}\right) V_{0}\left(n_{b}, T\right),
$$

where $N_{B}$ is the total number of nearest-neighbor pairs in the whole system. 
Here, $\Omega\left(n_{b}\right)$ is the DOS for the bond number defined as the number of consistent combinations of graphs and states such that the graph consists of $n_{b}$ bonds. Then, the canonical average of a quantity $A$ is calculated by

$$
\langle A\rangle_{T}=\frac{\sum_{n_{b}}\langle A\rangle_{n_{b}} \Omega\left(n_{b}\right) V_{0}\left(n_{b}, T\right)}{Z(T)},
$$

where $\langle A\rangle_{n_{b}}$ is the microcanonical average with the fixed bond number $n_{b}$ for the quantity $A$. Thus, if we obtain $\Omega\left(n_{b}\right)$ and $\langle\cdots\rangle_{n_{b}}$ during the simulation process, we can calculate the canonical average of any quantity.

In deriving the BHR for the bond number, we follow a method similar to that used to derive the BHR for the bond number $[22,23]$. Instead of using the relation between states, we consider the relation between graphs. The number of potential moves from the graph with the bond number $n_{b}$ to the graph with $n_{b}+1, N\left(S, G, n_{b} \rightarrow n_{b}+1\right)$, for fixed $S$ is equal to that of the number of potential moves from the graph with $n_{b}+1$ to that with $n_{b}, N\left(S, G^{\prime}, n_{b}+\right.$ $\left.1 \rightarrow n_{b}\right)$. Taking a summation over states $S$ and using the definition of the microcanonical average with the fixed bond number $n_{b}$, we have

$$
\Omega\left(n_{b}\right)\left\langle N\left(G, n_{b} \rightarrow n_{b}+1\right)\right\rangle_{n_{b}}=\Omega\left(n_{b}+1\right)\left\langle N\left(G^{\prime}, n_{b}+1 \rightarrow n_{b}\right)\right\rangle_{n_{b}+1} .
$$

This is the BHR for the bond number. It should be noted that $N\left(G, n_{b} \rightarrow\right.$ $\left.n_{b}+1\right)$ is a possible number of bonds to add, and related to the number of satisfied pairs for the given state $S, n_{p}(S)$, by $N\left(G, n_{b} \rightarrow n_{b}+1\right)=n_{p}(S)-n_{b}$. With use of the microcanonical average with fixed bond number for $n_{p}$, we have the relation

$$
\left\langle N\left(G, n_{b} \rightarrow n_{b}+1\right)\right\rangle_{n_{b}}=\left\langle n_{p}\right\rangle_{n_{b}}-n_{b}
$$

On the other hand, the possible number of bonds to delete, $N\left(G^{\prime}, n_{b}+1 \rightarrow n_{b}\right)$, is simply given by $n_{b}+1$, that is,

$$
\left\langle N\left(G^{\prime}, n_{b}+1 \rightarrow n_{b}\right)\right\rangle_{n_{b}+1}=n_{b}+1
$$

From the BHR for the bond number, Eq. (15), we have

$$
\frac{\Omega\left(n_{b}\right)}{\Omega(0)}=\prod_{l=0}^{n_{b}-1} \frac{\langle N(G, l \rightarrow l+1)\rangle_{n_{b}=l}}{\langle N(G, l+1 \rightarrow l)\rangle_{n_{b}=l+1}}
$$

Then, substituting Eqs. (16) and (17) into Eq. (18), we obtain the bond- 
number DOS, $\Omega\left(n_{b}\right)$, as

$$
\ln \Omega\left(n_{b}\right)=\ln \Omega(0)+\sum_{l=0}^{n_{b}-1} \ln \left(\frac{\left\langle n_{p}\right\rangle_{n_{b}=l}-l}{l+1} .\right) .
$$

When calculating the bond-number DOS from the BHR for the bond number, we only need the information on $\left\langle n_{p}\right\rangle_{n_{b}}$, the microcanonical average with fixed $n_{b}$ of the number of satisfied pairs $n_{p}$. It is much simpler than the case of the BHR formulation for the energy DOS.

Moreover, in the computation of $n_{p}$, we can use an improved estimator. If a pair of sites $(i, j)$ belong to the different cluster, this pair is satisfied with a probability of $1 / Q$. If a pair of sites belong to the same cluster, this pair is always satisfied. Then, we can employ an improved estimator $\tilde{n}_{p}$ as

$$
\tilde{n}_{p}(G)=\left(1-\frac{1}{Q}\right) \sum_{\langle i, j\rangle} \delta_{c_{i}(G), c_{j}(G)}+\frac{N_{B}}{Q},
$$

where $c_{i}(G)$ represent a cluster that a site $i$ belongs to. Only the information on graph is needed.

Let us consider the update process for the Monte Carlo simulation. In the multibondic ensemble method, a graph is updated by adding or deleting a bond for a satisfied pair of sites based on $\Omega\left(n_{b}\right)$ [14]. We may use the number of potential move for the bond number, $\langle N(G, \cdots)\rangle_{n_{b}}$, for the probability of update. Using Eqs. (15), (16), and (17), we get the probability to delete a bond,

$$
P\left(n_{b} \rightarrow n_{b}-1\right)=\frac{\left\langle n_{p}\right\rangle_{n_{b}-1}+1-n_{b}}{\left\langle n_{p}\right\rangle_{n_{b}-1}+1},
$$

and the probability to add a bond,

$$
P\left(n_{b} \rightarrow n_{b}+1\right)=\frac{n_{b}+1}{\left\langle n_{p}\right\rangle_{n_{b}}+1}
$$

respectively.

The actual Monte Carlo procedure is as follows. We start from some state (spin configuration) $S$, and an arbitrary graph $G$ consistent with it. We add or delete a bond of satisfied pairs with the probability (21) or (22). After making such a process as many as the number of total pairs, $N_{B}$, we flip every cluster with the probability $1 / 2$. And we repeat the process. Since we do not 
know the exact form of $\left\langle n_{p}\right\rangle_{n_{b}}$, we use the accumulated average for $\left\langle n_{p}\right\rangle_{n_{b}}$. The dynamics proposed here can be regarded as the flat histogram method for the bond number, which we call the cluster-flip flat histogram method. As $\left\langle n_{p}\right\rangle_{n_{b}}$ converges to the exact value, the histogram $H\left(n_{b}\right)$ becomes flat. We calculate the bond-number DOS, and then calculate various quantities by Eq. (14).

As a test, we calculate the $L \times L \times L$ Ising model on the simple cubic lattice with the periodic boundary conditions by using the cluster-flip flat histogram method. We show $\left\langle n_{p}\right\rangle_{n_{b}} / N_{B}$ as a function of $n_{b}$ for $L=12$ by the solid line in Fig. 3(a); we give $n_{b} / N_{B}$ by the dotted line. The number of Monte Carlo sweeps (MCS) is $5 \times 10^{7}$. The difference between the solid and dotted lines represents the number of potential moves $\left\langle N\left(n_{b} \rightarrow n_{b}+1\right)\right\rangle / N_{B}$, whereas the difference between the dotted line and the horizontal axis represents $\left\langle N\left(n_{b} \rightarrow n_{b}-1\right)\right\rangle / N_{B}$. We note that $\left\langle n_{p}\right\rangle_{n_{b}=0} / N_{B}=1 / 2$, which is expected from Eq. (20). The logarithm of the bond-number DOS, $\ln \Omega\left(n_{b}\right)$, obtained by $\left\langle n_{p}\right\rangle_{n_{b}}$ is shown in Fig. 3(b) as a function of $n_{b}$. The temperature dependence of the specific heat is shown in Fig. 3(c), which reproduces the result obtained by the conventional method.
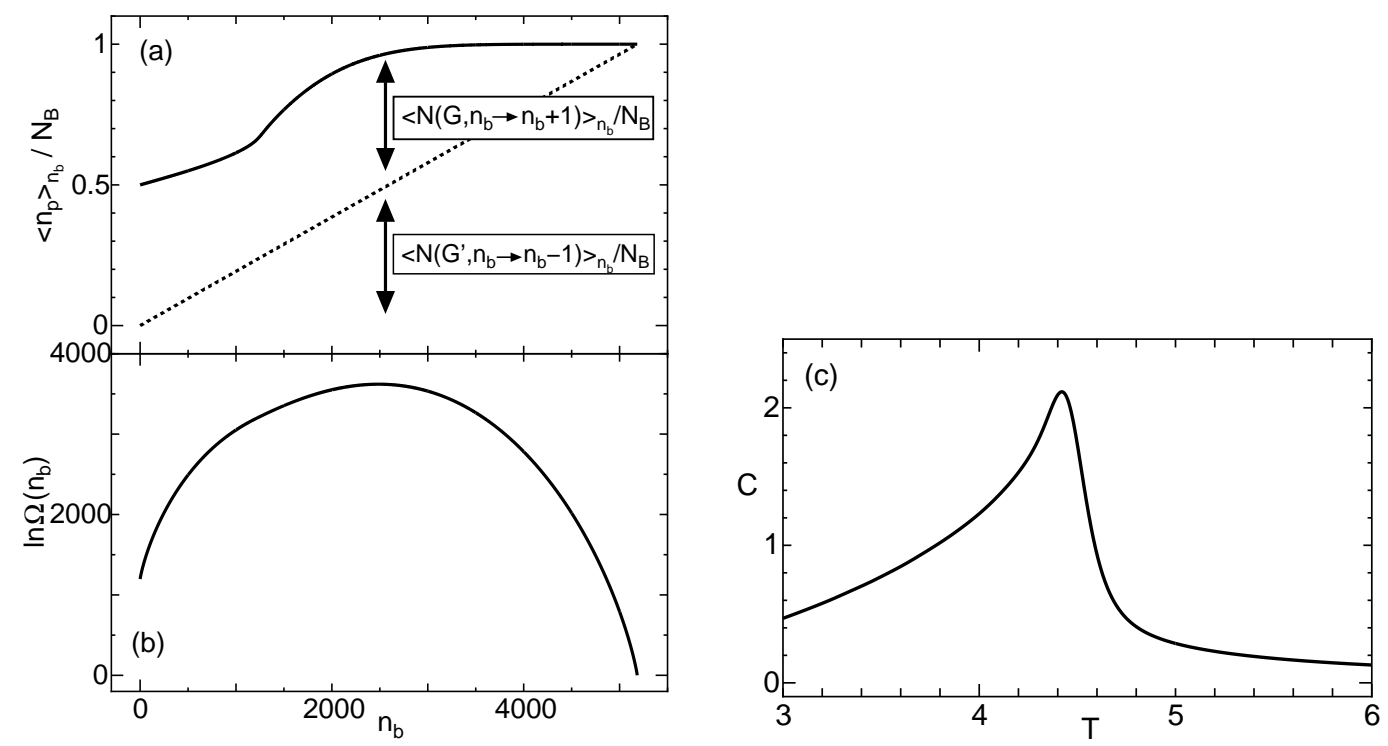

Fig. 3. (a) $\left\langle n_{p}\right\rangle_{n_{b}} / N_{B}$ and (b) $\ln \Omega\left(n_{b}\right)$ of the $12 \times 12 \times 12$ Ising model obtained by the cluster-flip flat histogram method. The dotted line in (a) denotes $n_{b} / N_{B}$. (c) The temperature dependence of the specific heat per spin, where we have used the units of $J=k_{B}=1$.

As another example, we simulate the 3D 3-state Potts model on the simple cubic lattice. A first-order phase transition occurs in this model. We show $\left\langle n_{p}\right\rangle_{n_{b}} / N_{B}$ for $L=12$ by the solid line in Fig. 4(a); we give $n_{b}$ by the dotted line. The number of MCS is $5 \times 10^{7}$. The number of potential moves $\left\langle N\left(n_{b} \rightarrow n_{b}+1\right)\right\rangle / N_{B}$ and $\left\langle N\left(n_{b} \rightarrow n_{b}-1\right)\right\rangle / N_{B}$ are given in the same manner as the case of the Ising model. It is to be noted that $\left\langle n_{p}\right\rangle_{n_{b}=0} / N_{B}=1 / 3$ for the 3 -state Potts model. The logarithm of the bond-number DOS, $\ln \Omega\left(n_{b}\right)$, 
obtained by $\left\langle n_{p}\right\rangle_{n_{b}}$ is shown in Fig. 4(b). The temperature dependence of the free energy is given in Fig. 4(c). The first-order point where the derivative of the free energy has a jump is indicated by the arrow.
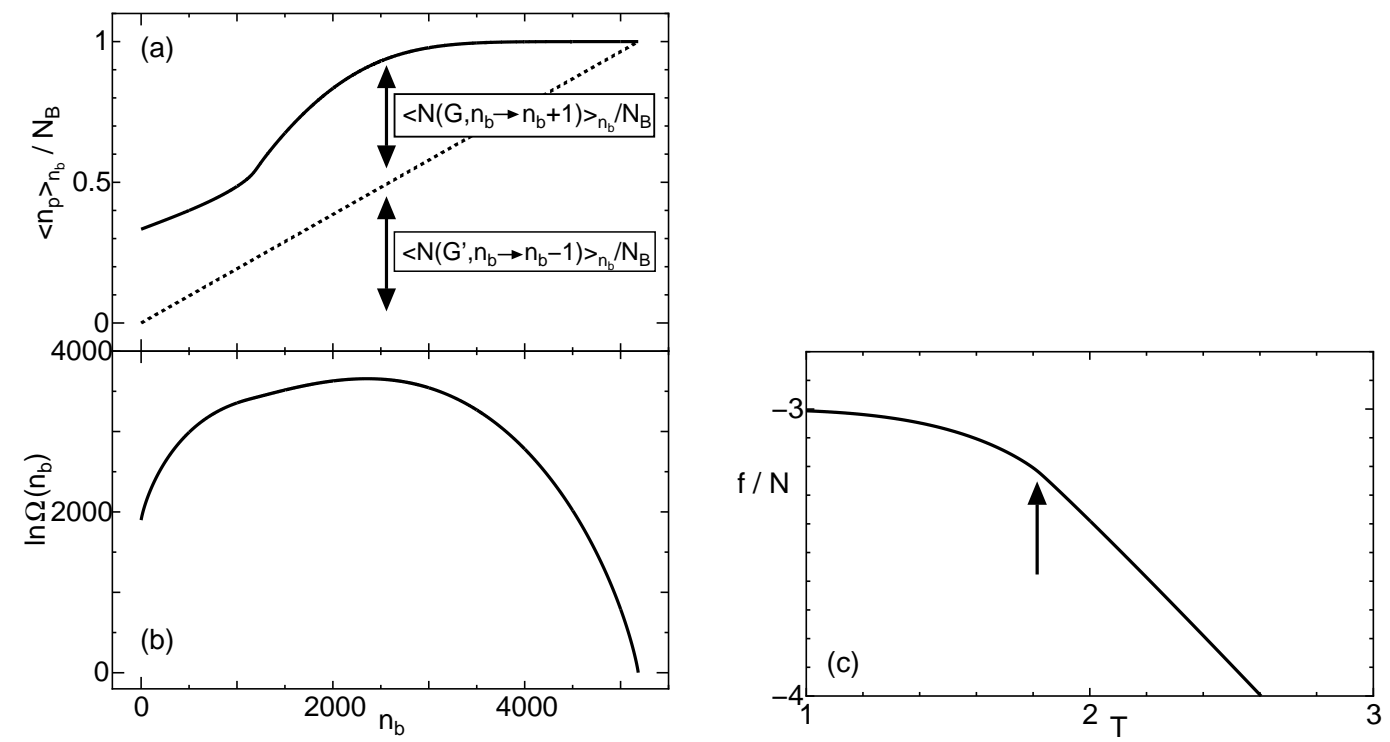

Fig. 4. (a) $\left\langle n_{p}\right\rangle_{n_{b}} / N_{B}$ and (b) $\ln \Omega\left(n_{b}\right)$ of the 3D 3-state Potts model of $L=12$ obtained by the cluster-flip flat histogram method. The dotted line in (a) denotes $n_{b} / N_{B}$. (c) The temperature dependence of the free energy per spin, where we have used the units of $J=k_{B}=1$.

The detailed report on the subject of this section will be published in a separate paper [26]. There, the results for the application to the 2D Ising and 10-state Potts models will be given, and the efficiency of the method will be discussed.

\section{Summary and discussions}

We have discussed the recent progress in Monte Carlo algorithms. First, we have argued the generalization of the PCC algorithm based on the study of the FSS property of the correlation ratio, the ratio of the correlation functions with different distances. We apply this generalized scheme of the PCC algorithm to the 2D 6-state clock model. Since we do not use the percolating property of the system, we can apply the PCC algorithm where the mapping to the cluster formalism does not exist. It can be applied to many problems. For example, the cluster formalism does not work well for frustrated systems, but we can use the generalized PCC algorithm. We can also apply the generalized scheme to a wide variety of quantum systems.

Second, we have discussed the combination of the cluster algorithm and the extended ensemble method. We have derived the rigorous BHR for the bond 
number, investigating the cluster (graph) representation of the spin models. We have shown that the bond-number DOS $\Omega\left(n_{b}\right)$ can be calculated in terms

of $\left\langle n_{p}\right\rangle_{n_{b}}$. We have proposed a Monte Carlo dynamics based on the number of potential moves for the bond number, which is regarded as the flat histogram method for the bond number. We have applied the cluster-flip flat histogram method to the 3D Ising and 3-state Potts models.

\section{Acknowledgments}

We thank N. Kawashima for fruitful discussions and the collaboration of a part of the present work. We also thank H. Otsuka, J.-S. Wang and C.-K. Hu for valuable discussions. The computation in this work has been done using the facilities of the Supercomputer Center, Institute for Solid State Physics, University of Tokyo. This work was supported by a Grant-in-Aid for Scientific Research from the Ministry of Education, Science, Sports and Culture, Japan.

\section{References}

[1] R. H. Swendsen and J. S. Wang, Phys. Rev. Lett. 58, 86 (1987).

[2] U. Wolff, Phys. Rev. Lett. 62, 361 (1989).

[3] B. A. Berg and T. Neuhaus, Phys. Lett. B 267, 249 (1991); Phys. Rev. Lett. 68, 9 (1992).

[4] J. Lee, Phys. Rev. Lett. 71, 211 (1993).

[5] P. M. C. de Oliveira, T. J. P. Penna, and H. J. Herrmann, Braz. J. Phys. 26, 677 (1996); Eur. Phys. J. B 1, 205 (1998).

[6] J.-S. Wang, Eur. Phys. J. B 8, 287 (1998).

[7] J. S. Wang and L. W. Lee, Comp. Phys. Commun. 127, 131 (2000); J. S. Wang, Physica A 281, 147 (2000).

[8] F. Wang and D. P. Landau, Phys. Rev. Lett. 86, 2050 (2001); Phys. Rev. E 64, 056101 (2001).

[9] C. Yamaguchi and Y. Okabe, J. Phys. A 34, 8781 (2001).

[10] Y. Tomita and Y. Okabe, Phys. Rev. Lett. 86, 572 (2001); J. Phys. Soc. Jpn. 71, 1570 (2002).

[11] Y. Tomita and Y. Okabe, Phys. Rev. E 64, 036114 (2001).

[12] Y. Tomita and Y. Okabe, Phys. Rev. B 65, 184405 (2002). 
[13] J. Kosterlitz and D. Thouless, J. Phys. C 6, 1181 (1973).

[14] W. Janke and S. Kappler, Phys. Rev. Lett. 74, 212 (1995).

[15] C. Yamaguchi and N. Kawashima, Phys. Rev. E 65, 056710 (2002).

[16] P. W. Kasteleyn and C. M. Fortuin, J. Phys. Soc. Jpn. Suppl. 26, 11 (1969); C. M. Fortuin and P. W. Kasteleyn, Physica 57, 536 (1972).

[17] C.-K. Hu, Phys. Rev. B 29, 5103 and 5109 (1984).

[18] C.-K. Hu, Phys. Rev. B 46, 6592 (1992); Phys. Rev. Lett. 69, 2739.

[19] K. Binder, Z. Phys. B 43, 119 (1981).

[20] J. V. José, L. P. Kadanoff, S. Kirkpatrick, and D. R. Nelson, Phys. Rev. B 16, 1217 (1977).

[21] Y. Tomita and Y. Okabe, to appear in Phys. Rev. B.

[22] P. M. C. de Oliveira, Eur. Phys. J. B 6, 111 (1998).

[23] B. A. Berg and U. H. E. Hansmann, Eur. Phys. J. B 6, 395 (1998).

[24] D. Kandel and E. Domany, Phys. Rev. B 43, 8539 (1991).

[25] N. Kawashima and J. E. Gubernatis, Phys. Rev. E 51, 1547 (1995).

[26] C. Yamaguchi, N. Kawashima, and Y. Okabe, Phys. Rev. E. 66, 036704 (2002). 